\title{
Analyzing Business Models for Mobile Augmented Reality
}

\author{
Ana M. Bernardos, José R. Casar \\ Universidad Politéenica de Madrid \\ Telecommunications School \\ Madrid, Spain \\ abernardos@grpss.ssr.upm.es
}

\begin{abstract}
Nowadays, a wide offer of mobile augmented reality (mAR) applications is available at the market, and the user base of mobile AR-capable devices -smartphones- is rapidly increasing. Nevertheless, likewise to what happens in other mobile segments, business models to put $m A R$ in value are not clearly defined yet. In this paper, we focus on sketching the big picture of the commercial offer of $m A R$ applications, in order to inspire a posterior analysis of business models that may successfully support the evolution of mAR. We have gathered more than $400 \mathrm{mAR}$ applications from Android Market, and analyzed the offer as a whole, taking into account some technology aspects, pricing schemes and user adoption factors. Results show, for example, that application providers are not expecting to generate revenues per direct download, although they are producing high-quality applications, well rated by the users.
\end{abstract}

Augmented reality; business models; mobile applications

\section{INTRODUCTION}

'Augmented reality' (AR) is not a new concept: the term, as it is, is in use since 1992 [1], but it has been revisited since the sixties, when Sutherland [2] designed a head-mounted display tracked by both mechanical and ultrasonic sensors. Basically, augmented reality relies on combining and superimposing virtual information over the real world, providing the user with extra (even real time) computer-based information about spatial resources.

Nowadays, the generalization of high-resolution cameras, big screens, embedded compasses and inertial systems in mobile devices has created the technological breeding ground for the democratization of (limited but reasonable) mobile $A R$ (mAR) immersive experiences. The number of AR-capable mobile devices is quickly rising - e.g. [3] states that the number of smartphones increased up to be $19 \%$ of the mobile phones sold in 2010, 72,1\% more than in 2009. Nevertheless AR is still unknown for most of mobile users, even if manufacturers such as Samsung or LG have decided to include preinstalled AR applications (e.g. Layar) in their devices to spread user's awareness.

Anyhow, forecast figures estimate at $\$ 1.5-2.2$ billion the revenues generated by $\mathrm{mAR}$ [4][5] by 2015, while other analy sts claim that the technology is still overhyped [6].
In spite of user adoption related issues and differing opinions with respect to the technology's interest, the commercial offering of mobile AR applications is increasingly broadening. At a glance, the ecosystem of available mobile AR applications is nowadays diverse. For example, it is possible to find a wide variety of:

- Generic AR browsers, which allow screening points of interest (any type) in the area of presence. E.g. Layar, Wikitude.

- Guides, focused on providing complex information in an accessible way. Some of them include user generated contents and social networking. E.g. LP Compass, buUuk, junaio.

- Search engines, which facilitate finding the right information in the environment. E.g. Google Goggles, Golf Range Finder, Food Tracer, SREng, Cyclopedia, AR Compass.

- Augmented navigation, to enhance the experience of going from one point to another or finding the way to a location-tagged resource. E.g. Nearest Subway, Augmented Car Finder, SpyGlass, Theodolite.

- Object-superimposing based applications, mainly focused on décor, art or fashion. They make possible to discover virtual objects indoors and outdoors and place them over a body or in a specific place of a room. E.g. See It On, The Public Virtual Art Project, Snap Shot Showroom.

- Time machines, still on prototype in their most advanced versions, serve to show how a place was in the past or how it will be in the future. E.g. iTacitus, StreetMuseum.

- Games, both marker-based and sensor based. E.g. AR Basket, TagDis, SpecTrec, ARDefender, SkySiege, SpaceInvadAR, Parallel Kingdom, Rock'em Sock'em.

- Skymaps and weather applications. They are conceived to explore virtual information (satellite position, direction of the breeze, etc.) to be displayed in the sky. E.g. Skymap, Sunseeker, SeeBreezer, Dishpointer, Meteo360. 
- Tools for disability, in particular to allow color-blind individuals to cope with the chromatic scale. Eg. Dankam, Chromatic Glass.

- Translators, which makes possible to see real signs in different languages. E.g. Word Lens.

- People recognizers, capable of discriminating physical traits of individuals (mainly face). E.g. Recognizr, Wiewdle.

Business models supporting these applications and services are still undefined or unclear in most of cases. In order to prepare a subsequent analysis of the different factors that may affect mAR business models, this paper reviews some general aspects that describe the commercial offer of mobile augmented reality applications. To do so, we have collected data from the second most popular marketplace of mobile applications, Android Market. Our methodology is described in Section II. Sections III and IV present the results of our analy sis, while Section V gathers some conclusions. We aim at describing mAR commercial applications from different points of view: functional offer, technology use, pricing schemes and user adoption, but basing on an extensive and updated database, as we feel that this is the fist step for a subsequent and in-depth analysis of the business models supporting individual applications.

\section{ANALYZING THE OFFERING OF COMMERCIAL MAR APPLICATIONS: METHODOLOGY}

In order to systematize the analysis of the current market of $\mathrm{mAR}$ applications, we have gathered in a structured database the offering available in Android Market during June-July 2011, retrieving a total of 442 applications. Android Market, available since October 2008, is an online software store developed by Google for Android phones.

Nowadays, application stores dominate across application distribution channels [7]; application providers intensively rely on them to deliver their products to the market, so these virtual stores can be considered as adequate information sources to describe the available commercial offer. Apple Store is the biggest marketplace in number of applications, followed by Android Market (aprox. 350.000 applications vs. 200.000 in Q1 2011 [8]), but some studies state the rapid growing pace of the latter [7]. Choosing Android Market instead of Apple Store, Nokia Ovi Store, Blackberry App World, Windows Marketplace or any other independent store has been a decision in the search of equilibrium between diversity (every significant application in terms of functionality is very likely to be delivered for Android phones) and information volume. When considering the quality of data, it is important to note that Google publishing strategy allows developers to upload, update and publish applications directly (no approval procedure is needed), so application providers are directly providing most part of the information in the database. Inaccuracies may exist, as e.g. criteria for classification may differ from an individual to other.

The selection of applications for our database has been done using the term 'augmented reality' as keyword for search in the English version of Android Market (the number of applications slightly vary depending on the website language). Of course, this decision may have biased the final result, excluding some applications, but every application included in the database provides augmented reality features.

From the information available at Android Market, we have extracted the following fields for the database:

- General information: application provider, category, last update date and short description. Categories for classification are the ones proposed in Android Market: 22 general categories (e.g. communications, finance, health\&fitness, lifestyle, news\&magazines, productivity, travel\&local, personalization, etc.) and 5 game categories (for the complete list, see [9]).

- Technology information: from the basic information available in the Market, it is possible to retrieve the Android version on which the application runs and its memory size.

- Business information: target market (derived from content rating) and pricing scheme. Content rating provides information on the market focus depending on the age (Everyone, and Low, Medium, High Maturity [10]).

- User adoption information: it is shown through the number of installs and the rating (average satisfaction vs. number of opinions) that users are giving back

Additionally, Android Market provides information on which type of permissions are needed for an application to work. The list of permissions that have been identified for mAR applications is in Table 1, classified by the general type of resources that need to be used. Information about permissions makes possible to analyze, with some limitations: a) some additional aspects of the application's technical needs, taking into account the underlying technologies that enable $\mathrm{mAR}$ and b) information about the use of personal data, an aspect that may be related, on one hand, to the application's adaptation and personalization capabilities and, on the other hand, to privacy aspects [11].

With respect to technical aspects, the list of permissions provides information on the use of positioning technologies and video control. In brief, we could say that $A R$ works on recognizing objects, scenes and relative positions. In order to calculate the spatial relationship between a user (carrying a visualization display) and a reference point (object or location), three types of techniques are usually applied (interested readers in AR tracking technology can resort to e.g. [12]): vision-based tracking (based on image recognition), sensor-based tracking (working on inertial and positioning data) and hybrid tracking (combining both methods). The first group of techniques relies on the use of a camera to continuously 'snapshot' the target object (a marker) and process the image to estimate the position, orientation and movement of the visualization display with respect to the target object. The second group of techniques works with algorithms that fuse orientation, movement and location estimates from inertial and positioning systems to physically reference the user to the target object. When outdoors, GPS offers sufficient accuracy to locate the 
user, while compasses may provides orientation and accelerometers, magnetometers and gyros allow estimating the device's relative inclination. When indoors, it is necessary to switch to costly and dense infrastructures (e.g. based on ultrawideband, ultrasounds or infrared localization systems), typically complemented with user-worn devices, in order to achieve centimeter-level accuracy, or to rely in position markers from which the user is able to explore the environment. From the collected data, it will not be possible to accurately analyze the type of technology that applications use to enable augmented reality, but to have a hint on the percentage of applications using sensor-based or vision-based techniques.

TABLE I. LIST OF PERMISSIONS ON THE MOBILE DEVICE

\begin{tabular}{|c|c|}
\hline Type of permission & Requirement \\
\hline \multirow[t]{2}{*}{ Accounts } & Use the authentication credentials of an account \\
\hline & Manage the accounts list \\
\hline \multirow{3}{*}{ Hardware controls } & Take pictures and videos \\
\hline & Record sound \\
\hline & Change your audio settings \\
\hline \multirow{3}{*}{ Location } & Fine (GPS) Location \\
\hline & Coarse (network-based) location \\
\hline & Mock location sources for testing \\
\hline \multirow{2}{*}{$\begin{array}{l}\text { Network } \\
\text { communications }\end{array}$} & Create Bluetooth connections \\
\hline & Full Internet Access \\
\hline \multirow[t]{2}{*}{ Phone calls } & Read phone state and identity \\
\hline & Intercept outgoing calls \\
\hline \multirow[t]{2}{*}{ Storage } & Modify/delete USB storage contents \\
\hline & Modify/delete SD Card Contents \\
\hline \multirow{9}{*}{ System tools } & Prevent device from sleeping \\
\hline & Bluetooth administration \\
\hline & Make application always run \\
\hline & Change state of $\mathrm{WiFi}$ \\
\hline & Change your UI settings \\
\hline & Modify the global settings of the system \\
\hline & Change network connectivity \\
\hline & Mount and unmount file systems \\
\hline & Retrieve running applications \\
\hline \multirow{5}{*}{ Personal information } & Read calendar events \\
\hline & $\begin{array}{l}\text { Add or modify calendar events and send email to } \\
\text { guests }\end{array}$ \\
\hline & Read contact data \\
\hline & Write contact data \\
\hline & Read browser's history and bookmarks \\
\hline \multirow[t]{3}{*}{ Messaging } & Read SMS or MMS \\
\hline & Receive SMS \\
\hline & Edit SMS or MMS \\
\hline \multirow{2}{*}{$\begin{array}{l}\text { Services that cost } \\
\text { money }\end{array}$} & Send SMS messages \\
\hline & Directly call phone numbers \\
\hline
\end{tabular}

With respect to the use of personal information, we will consider that accessing data of accounts, location, phone state and outgoing calls, running applications, calendar and contact list may increase the adaptation and personalization capabilities of mAR applications. If data are transmitted over any type of communication network, this intensive use of personal data may have privacy implications.

The following sections gather a preliminary analysis of the collected data, firstly focused on describing the functionalities and enabling technologies used by $\mathrm{mAR}$ applications, and secondly gathering information about their pricing strategies and user adoption features.

\section{THE MAR ECOSYSTEM: GENERALITIES AND TECHNICAL ASPECTS}

\section{A. Generalities}

In a month time (from June 13rd to July 8th), the number of applications obtained by using 'augmented reality' as search keywords has increased from 422 to 522 , that is to say $24 \%$. This fact shows the lively pace of introduction of new $\mathrm{mAR}$ applications into the market of Android phones. $\mathrm{mAR}$ applications are a small portion of the whole offer, around $0,13 \%$.

The analysis of the offering by category (Fig. 1) shows that travel\&local clearly gathers the most significant share of mAR applications (38\%): city guides, local business information or trip management tools are enhanced through augmented reality features or created to fully exploit the mAR concept. Far behind, entertainment, lifestyle and tools categories group $24 \%$ of $\mathrm{mAR}$ applications. Social and arcade\&action games represent $5 \%$ over the total, being the rest of applications very segmented into diverse functionalities, which include e.g. sports, transportation or productivity.

From the content rating data, it is possible to infer that $\mathrm{mAR}$ applications are focusing on the general market $(75 \%$ of applications are for everyone or 'low mature' clients), although it is not feasible any further market segmentation relying on this information.

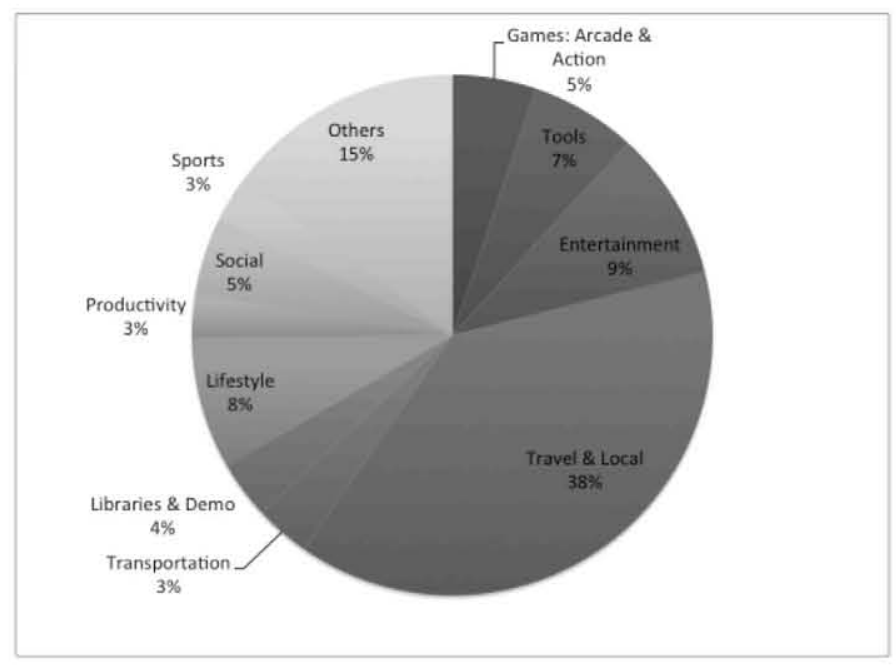

Figure 1. mAR applications by category.

\section{B. Technical aspects}

Android operating system's tools facilitate to sensors and camera features needed to implement $\mathrm{mAR}$ performance from its very first versions. Nevertheless, subsequent releases have enhanced its functionalities regarding positioning and camera access. In Fig. 2 there is a comparison between the versions of the operating systems for which the mAR offering is optimized (Fig. 2a), against the installed user base (Fig. 2b).

Data from Google [13] state that most mobile devices accessing Android Market in July 2011 (59,6\%) were using 
Android 2.2 'Froyo' (released on May 2010), while 19\% of the $\mathrm{mAR}$ applications stored in our database have been designed for this version. Only $1,4 \%$ of mobile devices had Android 1.5 'Cupcake' (released on April 2009) installed, while the majority of mAR applications (34\%) have been implemented to work on this version. Regarding enabling technologies for mAR (positioning and vision-based features), version 1.6 'Donut' (Sept. 2009) includes speed improvements in camera applications. Version 2.0 / 2.1 'Eclair' (Oct. 2009, Jan. 2010) provides optimized hardware speed and better management of display functions. After 'Cupcake', mAR developers prefer to use one of these two versions $(24 \%$ choses 'Donut', while $25 \%$ use 'Eclair'). Android 2.3 'Gingerbread' (released on Dec. 2010) provides access to multiple cameras, NFC support and virtual sensors to facilitate accessing gravity, acceleration and rotation, but no $\mathrm{mAR}$ application has been specifically designed for it. The same happens with 3.x 'Honeycomb' (released on Feb. 2011).
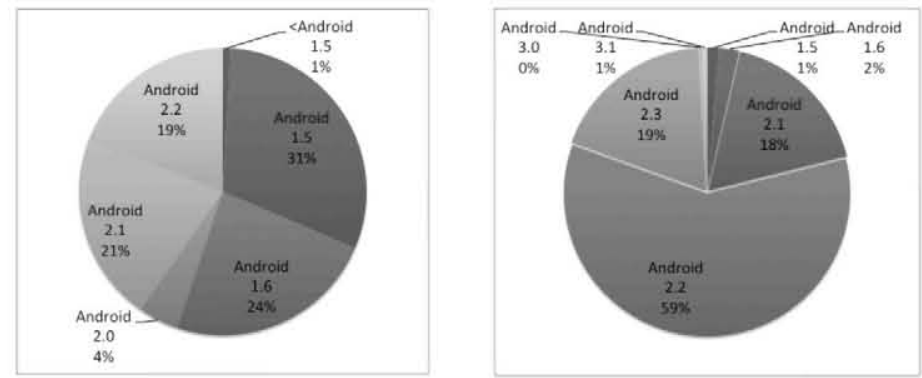

Figure 2. a) $\mathrm{mAR}$ applications by Android version; b) installed Android user base in July 2011 (data source: Google [13]). The memory fingerprint of mAR applications is in most of cases smaller than $1 \mathrm{MB}(56 \%)$, and almost every case, smaller than $10 \mathrm{MB}(96 \%)$.

When analzying the device's resources that mobile AR applications need to access (Fig. 3), it happens - as expected that most of them need to control hardware aspects or manage location information.

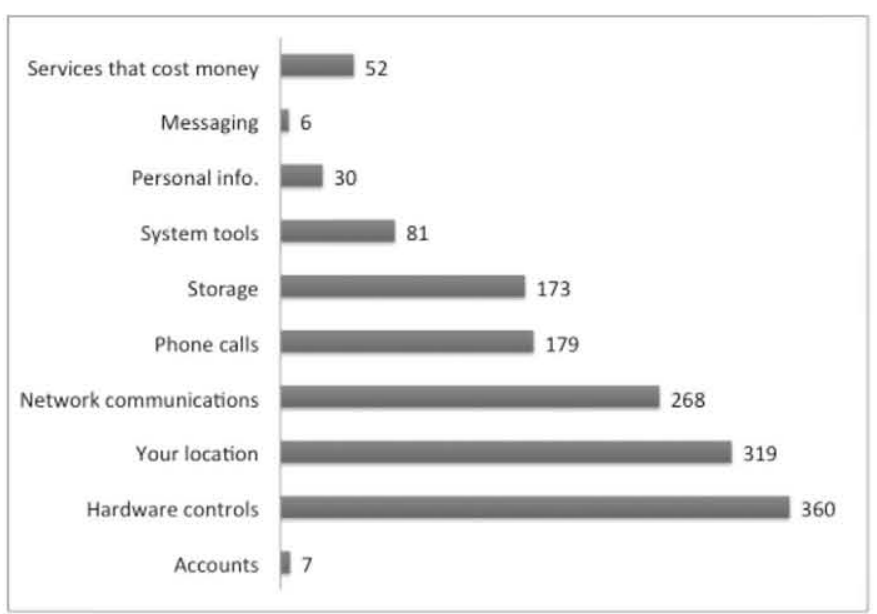

Figure 3. Permissions requested by $\mathrm{mAR}$ applications.

In particular, to have a view on the potential enabling technologies for $\mathrm{mAR}$, we have explored the permission requests to access different types of location technologies
(GPS or network-based) and the use of camera features. All the applications using location technologies (72\% over the total) access GPS; of those, 72\% additionally use coarse (network-based) location. Camera-based features are used by $81 \%$ of applications, while $84 \%$ of applications using GPS also ask for permission to handle camera-based features. Almost all travel\&local applications use GPS location (94\%) and access camera features (85\%).

When analyzing to which extent there are mAR applications just relying on image recognition, we have accounted that $14 \%$ of applications (60) enables access to camera features and do not use any type of location information: games, applications to remedy color-blindness, musical instruments or marketing tools are included in this group.

Some of the permissions requested by the applications might be related to personalization, as they access different type of user data in the device (calendar, contact data, running applications, outgoing calls, phone state, identity, location and accounts). Fig. 4 shows how many applications access each of these controls. From the chart, it can be noted that location management is the most relevant feature for $\mathrm{mAR}$.

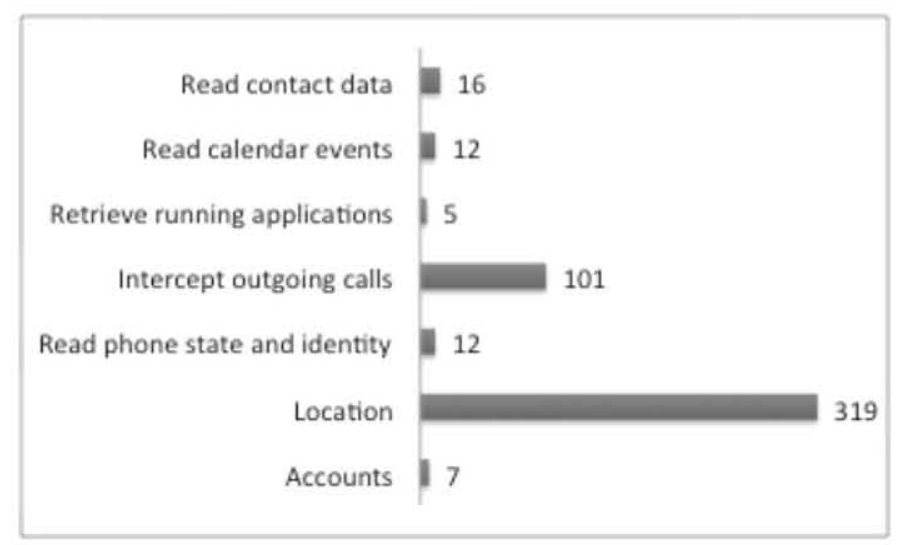

Figure 4. Use of resources that permit personalization.

\section{ON PRICING AND USER ADOPTION}

In this Section, a brief analysis on the pricing models for $\mathrm{mAR}$ is accomplished, taking into account user adoption factors, in the form of number of installs of mobile applications and user satisfaction (rating).

\section{A. Sketching the business model: pricing for $m A R$}

The collected data make possible to have a look at the pricing strategy of $\mathrm{mAR}$. From Fig. 5, it is possible to conclude that application providers seem not to focus on getting direct incomes from applications downloads, as $74 \%$ of applications are offered for free.

Seven percent costs less than one euro, while $12 \%$ are above 4 euros. These 'high-priced' applications are $\mathrm{mAR}$ travel guides (e.g. mTrip), mAR navigation assistants (e.g. Wikitude Drive, DashBoard Assist Pro), satellite finders (e.g. DispointerPro, Satellite Finder) and very specific applications for sports (phiGolf Voice Caddy). 


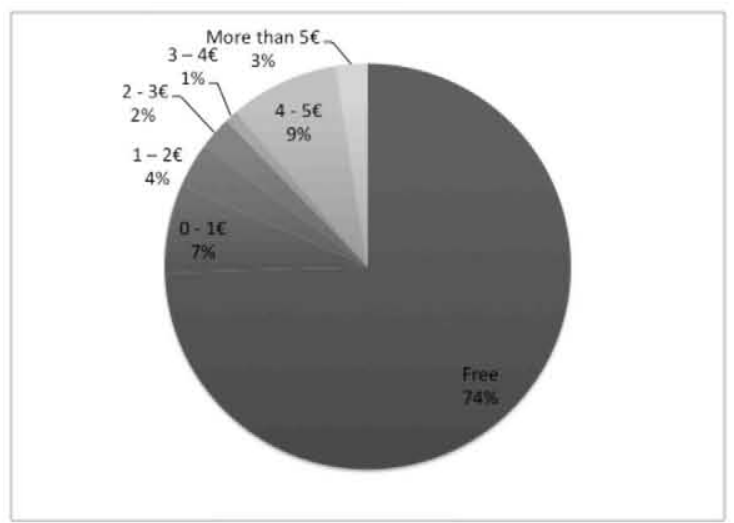

Figure 5. mAR applications by price.

\section{B. User adoption: notes on user acceptance}

Fig. 6 gathers the number of installs of mAR applications. Few of them $(7 \%)$ have been significantly downloaded (more than 50.000 installations). More than one third have been downloaded at least 1.000 times, while $57 \%$ still have a small user base. It is important to note that these results are not considering the 'application age', i.e. for how long the applications have been available in the marketplace.

There are three applications that have been downloaded more than 500.000 times: the AR browsers Layar and Wikitude (both released for Android in August 2009), and the navigation tool 3D Compass.

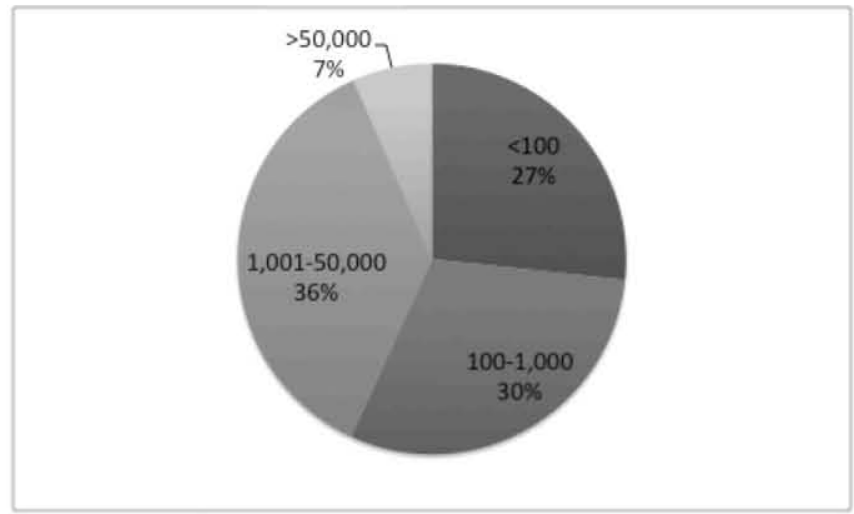

Figure 6. mAR applications by installs.

Fig. 7 shows the percentage of free/paid mAR applications that have been installed. Users are more attracted towards free applications: $55 \%$ of them have been installed more than 1.000 times, in comparison with $13 \%$ of paid applications. A recent report [14] analyzes this aspect for the total of applications in Android Market, concluding that $48 \%$ of free applications have been downloaded at least 1.000 times. When considering paid applications, we find a substantial difference: $79 \%$ of paid applications were downloaded less than 100 times, while in the case of mAR this percentage is reduced to $56 \%$.

When classified by category, we find that there are fewer paid applications than free ones (Fig. 8), except for transportation and travel\&local categories. Transportation includes applications offering notifications about traffic control, e.g. speed traps/cameras, school zones, etc. For travel\&local category, the number of paid/free applications is $78 / 92$.
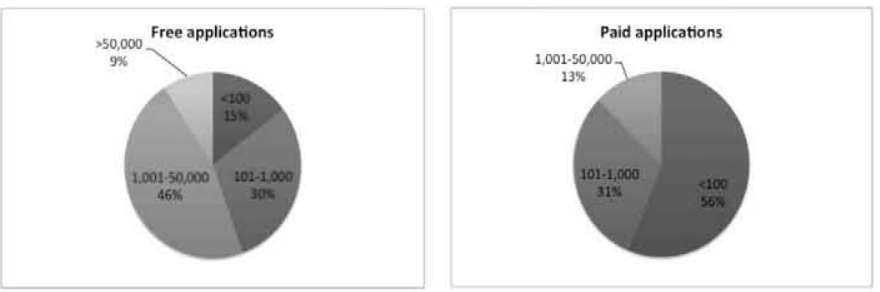

Figure 7. Free vs. paid $\mathrm{mAR}$ applications, by number of installs.

On a sample of the ' 100 most downloaded' applications (over 5.000 installs), travel\&local represent $26 \%$ over the total, followed by arcade\&action games, entertainment, tools and lifestyle applications ( $34 \%$ as a whole).

User evaluation is positive in this 'top 100' group: 87 of the most installed applications receive a user rating above 3 points (over 5), and 34 of them are above 4 points. The bestrated applications with a relevant number of opinions $(>400)$ are Locus Free (multi-function tourist application), Flightradar 24 Pro (application that shows airplane traffic around the world in real time) and Car Finder AR (which facilitates finding a parked car).

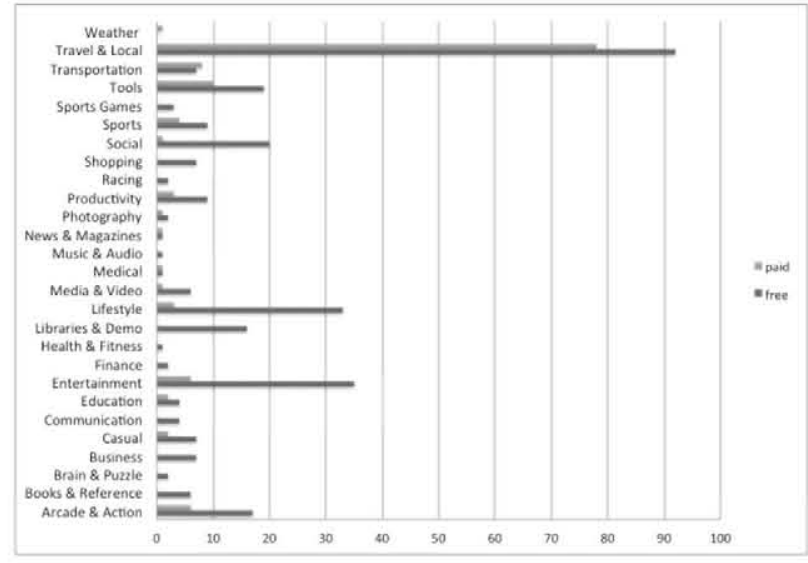

Figure 8. Free vs. paid applications, by category.

With respect to their pricing, in the 'top 100' group, 94 applications are free, while the rest are paid - prices are between 1,4-14€. The most costly application is Dish Pointer Pro (which uses AR to show the trajectories of satellites), and the cheapest, 3D Compass Pro. All the paid applications are rated with more than 4 points.

\section{CONCLUSIONS}

In this paper, we summarize an initial analysis of 422 mobile augmented reality applications released through Android Market. Data show the rapid generation pace of $\mathrm{mAR}$ applications, reinforcing the idea that $\mathrm{mAR}$ is, in practice, an attractive concept for application developers. In many cases, $\mathrm{mAR}$ features are included in already existent applications, which enhances their user experience through augmented 
reality. In particular, travel applications and those delivering information on local resources are leading both the offer of commercial mAR and the users' interest in terms of installs. These applications make an intensive use of location sensors and camera features.

Generally, mAR technical solutions seem to be based on sensor-based techniques, although many applications are accessing both fine-grained location sensors and camera controls. A limitation of our study is that technology aspects have not been inferred from technology-oriented parameters, but from 'permissions' they need to obtain to manage different resources in the mobile device. The catalogue of permissions is limited and ambiguous for our purposes in many cases. For example, it has been impossible to analyze the use of indoor location technologies for mAR: they may be related to permissions over communication networks such as $\mathrm{WiFi}$ or Bluetooth, but these controls are too general to extract any conclusion.

With respect to developer's and user device's OS, it seems that mAR applications are using more basic versions than the ones available at the installed base of Android devices. This may be due to the applications' age or to compatibility preferences of application providers. Although basic resources to enable $\mathrm{mAR}$ are available from early versions of Android, subsequent versions have provided complementary features regarding sensor access and camera management, so a priori, the use of newest OS versions may provide better final user experience.

Regarding the monetization strategy of mAR, we can state clear that most of the available applications are offered for free, so their providers are not expecting to get direct incomes from application downloads. It is possible that the business model for some of these free applications include strategies such as in-app advertising or indirect revenue shares on purchases initiated or motivated from the application use. Nevertheless, it is important to note that having chosen Android Market as source for data may influence this fact; i.e. the conclusion could be different if our research had been done on Apple Store. Apple Store and Android Market differ in their publication strategies, revenue shares and associated means of payments. Probably due to this latter issue, Apple Store is better performing on mobile applications commercialization.

$\mathrm{mAR}$ applications are succeeding among Android users when comparing their installation habits against those for nonmAR applications: the percentage of free mAR applications that have been installed at least 1.000 times is higher in the case of $m A R$. Additionally, $m A R$ paid applications have been installed more times on average than non-mAR ones.

When considering the most installed applications, both free and paid applications get good rating from users. In particular, all the paid applications in the 'top-100' list receive sound user evaluations, and two of the three most downloaded applications are paid ones.

From this initial study, further work will focus on performing an in-depth review of individual providers and applications; the latter will be filtered depending on the features previously considered. Our final objective is to study the business models that are successfully supporting $\mathrm{mAR}$, and compare them against others in the mobile ecosystem, in order to figure out if the particularities of mAR have any impact on the business side.

\section{ACKNOWLEDGMENT}

This work is being supported by the Government of Madrid under grant S2009/TIC-1485 and the Spanish Ministry for Science and Innovation under grant TIN2008-06742-C02-01. Authors acknowledge Paula Álvarez and Xinyue Chang (Data Processing and Simulation Group, Telecommunications School, UPM) their help to build the analysis database.

\section{REFERENCES}

[1] Caudell, T.P., Mizell, D.W., 1992, Augmented Reality: An Application of Heads-Up Display Technology to Manual Manufacturing Processes, Proceedings of 1992 IEEE Hawaii International Conference on Systems Sciences, pp. 659-669.

[2] Sutherland, I., 1968, A Head-Mounted Three Dimensional Display, Proceedings of Fall Joint Computer Conference, pp. 757-764.

[3] Gartner report, 2011, Competitive Landscape: Mobile Devices, Worldwide, 4Q10 and 2010. Partly available in: http://www.gartner.com/it/page.jsp?id=1543014 [accessed March 2011].

[4] ARChart, 2010, Mobile Phone Augmented Reality: Market Analysis and Forecasts. Partly available in: http://www.cellularnews.com/story/46677.php [accessed March 2011].

[5] Juniper Research, 2011, Mobile Augmented Reality. Partly available in: http://juniperresearch.com/viewpressrelease.php?pr=232 [accessed March 2011].

[6] Forrester Research, 2010, Mobile Augmented Reality. Partly available in: http://blogs.forrester.com/thomas_husson/10-12-20mobile_augmented_reality_beyond_the_hype_a_glimpse_into_the_mob ile future [accessed March 2011].

[7] Vision Mobile, 2011, Developer Economics: http://www.visionmobile.com/devecon.php [accessed July 2011].

[8] Business Insider, 2011, 'Charts of the Week, Google Is Closing The Gap On Apple's App Store': http://www.businessinsider.com/charts-of-theweek-ipad-competition-is-toast-201 1-3\#google-is-closing-the-gap-onapples-app-store-3\#ixzz1TEGoiFAn [accessed July 2011].

[9] Android market. Application categories: http://www.google.com/support/androidmarket/developer/bin/answer.py ?answer=113475 [accessed July 2011].

[10] Android market. Content rating: http:/www.google.com/support/androidmarket/developer/bin/answer.py ?\&\&answer $=188189$ [accessed July 2011].

[11] Bernardos, A.M., Casar, J.R., 2007, Building a framework to characterize location-based services, International Conference on Next Generation Mobile Applications, Services and Technologies NGMAST, pp.110-118.

[12] Feng, Z., Duh, H.B.-L., Billinghurst, M., 2008, Trends in augmented reality tracking, interaction and display: A review of ten years of ISMAR, 7th IEEE/ACM International Symposium on Mixed and Augmented Reality, pp. $193-202$.

[13] Platform versions http://developer.android.com/resources/dashboard/platformversions.html [accessed July 2011].

[14] Distimo, In-depth view on download volumes in the Google Android Market, May 2011, available at: http:/www.distimo.com/publications [accessed July 2011]. 